\title{
Insuficiência Cardíaca no Paciente Oncológico: Preditores de Risco
}

doi: https://doi.org/10.32635/2176-9745.RBC.2019v65n3.719

\author{
Heart Failure in Oncologic Patient: Risk Predictors \\ Insuficiencia Cardíaca en Pacientes con Cáncer: Predictores de Riesgo
}

Tatiana Abelin'; Marcos Jose Pereira Renni ${ }^{2}$

\section{INTRODUÇÃO}

Os avanços na detecção e tratamento do câncer e o envelhecimento da população levaram ao aumento do número de sobreviventes de câncer e continua a crescer anualmente ${ }^{1-4}$. Os efeitos do tratamento afetam a sobrevida e a qualidade de vida dos pacientes independente do seu prognóstico oncológico. A disfunção cardíaca relacionada à quimioterapia $(\mathrm{QT})$ é um dos efeitos indesejáveis e ocorre em cerca de $10 \%$ dos pacientes. A evolução para insuficiência cardíaca terminal acarreta um prognóstico de cerca de dois anos de sobrevida nesse período ${ }^{2}$.

A definição de cardiotoxicidade (CDTX) ainda gera discussão; mas, de acordo com os últimos consensos, ela é considerada quando se observa queda da fração de ejeção (FE) pelo ecocardiograma transtorácico (ECOTT) em mais de $10 \%$ do valor basal ou por sua queda para valores iguais ou abaixo de $53 \%{ }^{5}$.

Os agentes quimioterápicos causam ou exacerbam a insuficiência cardíaca por diversos mecanismos: por injúria miocárdica direta (antraciclinas, antimetablitos, agentes alquilantes, interleucinas-2); por exacerbação da hipertensão (inibidores dos fatores de crescimento endotelial); por facilitar a ocorrência de arritmias ou por outros mecanismos cardiotóxicos ainda não completamente compreendidos 5 . Pacientes com câncer ou sobreviventes de câncer expostos a esses agentes encontram-se sob maior risco de desenvolver insuficiência cardíaca ${ }^{3}$.

Apesar desses avanços, ainda estão em andamento estudos a respeito de marcadores precoces do desenvolvimento de CDTX precipitada por quimioterápicos diversos e principalmente pelas antraciclinas, as principais responsáveis pela lesão miocárdica permanente e causadora de desfechos negativos em médio e longo prazos. Do mesmo modo, ainda náo existe um escore de risco específico e universal que possa predizer com maior confiabilidade os pacientes mais suscetíveis ao desenvolvimento de insuficiência cardíaca secundária ao uso de quimioterápicos, apesar dos esforços das diversas sociedades envolvidas com a cardio-oncologia.

Sabe-se, no entanto, que a estratificação dos pacientes por meio da análise dos fatores de risco é um passo inicial na avaliação do paciente sujeito à CDTX. Aqueles mais suscetíveis a desenvolvê-la são os que recebem dose cumulativa maior do que $250 \mathrm{mg} / \mathrm{m}^{2}$, pacientes do sexo feminino, indivíduos com idade maior do que 65 anos e menores de 18 anos, portadores de insuficiência renal crônica, radioterapia $(\mathrm{RxT})$ mediastinal prévia ou concomitante ao tratamento, QT com agentes alquilantes ou antimicrotúbulos, além daqueles em uso concomitante de imunoterapias ${ }^{1,5}$.

Já os fatores de risco de desenvolvimento de CDTX para os que recebem quimioterápicos como ciclofosfamida, ifosfamida e taxanos (paclitaxel e docetaxel) são idade avançada, terapia combinada com outros antineoplásicos e irradiação mediastinal ${ }^{1}$.

Quanto àqueles que recebem imunoterapias, como inibidores de Her-2 (trastuzumabe e pertuzumabe) e inibidores de tirosina quinase (ITQ) como lapatinibe, possuem maior risco de evoluírem com CDTX quando seu uso está associado com agentes antimetabólitos e agentes alquilantes ${ }^{1}$. Interessante notar que mesmo pacientes mais jovens também apresentaram risco aumentado de desenvolver CDTX ${ }^{5}$. Pacientes com função global do ventrículo esquerdo (VE) próxima ao normal também estiveram mais suscetíveis ${ }^{1}$.

Diante desses dados e da avaliação dos fatores de risco, acrescentam-se ferramentas que têm se tornado cada vez mais presentes e fundamentais para a avaliaçáo do paciente submetido à QT por drogas com potencial cardiotóxico, incluindo dosagens de peptídeo natriurético tipo B (BNP), da troponina e o ECOTT acrescido da ferramenta strain, que avalia a deformação das fibras miocárdicas 5 .

O ECOTT é um método de importância fundamental nesse cenário, sendo um exame barato, sensível, além de

${ }^{1}$ Instituto Nacional de Câncer Jose Alencar Gomes da Silva (INCA). Rio de Janeiro (RJ), Brasil. Orcid iD: https://orcid.org/0000-0001-9093-7806 
amplamente disponível e não invasivo ${ }^{6}$. No entanto, o uso da FE ao ECOTT tem se mostrado pouco sensível para essa avaliação, surgindo novas técnicas mais inovadoras e sensíveis na predição de CDTX. Assim, durante um exame de ECOTT, os parâmetros de deformidade miocárdica por meio do strain 2D são utilizados para complementar os dados já habitualmente usados em um exame de rotina.

Os principais biomarcadores envolvidos na detecçáo da CDTX são as dosagens de troponina e do BNP/ proBNP. As troponinas são os marcadores padrão-ouro que diagnosticam a injúria miocárdica.

O BNP e a porção N-terminal do proBNP têm sido utilizados para a avaliação não invasiva da função sistólica do VE durante o tratamento com antineoplásicos. Os seus níveis séricos aumentados ocorrem em resposta à sobrecarga de pressão e volume e estão fortemente relacionados a eventos cardiovasculares, sintomas de insuficiência cardíaca e mortalidade ${ }^{7}$.

A troponina é outro importante marcador de detecção de lesão miocárdica de várias causas, como, por exemplo, infarto agudo do miocárdio, síndrome de Takotsubo e miocardite ${ }^{8}$. Medidas de troponina têm sido usadas em pacientes recebendo altas doses de antraciclínicos. A dosagem, por um método ultrassensível das troponinas 1 e $\mathrm{T}$, apresenta alta sensibilidade e especificidade como biomarcadores de dano miocárdico e suas concentraçôes estão associadas à severidade da injúria e ao desfecho clínico 9 . Estudo de Sawaya et al. ${ }^{10}$ demonstrou que o aumento da troponina associado com o strain longitudinal foram preditores da queda da FE.

Alguns serviços e sociedades propóem "escores de risco" ou fluxogramas específicos para estratificar o paciente com maior risco de desenvolver CDTX ${ }^{4}$. A maioria deles estratifica os fatores de risco tradicionais para o desenvolvimento de arteriosclerose e aqueles intrínsecos ao tratamento quimioterápico. É interessante notar que, apesar da sugestão de frequências maiores e menores de monitoramento para cada quimioterápico, os diversos serviços ressaltam a necessidade de um exame de base quando há previsão do uso de drogas cardiotóxicas independente da história clínica ${ }^{1}$. Questiona-se, porém, a viabilidade econômica de tal sugestão, já que todas as pacientes com câncer de mama, por exemplo, teriam que fazer ecocardiograma de base, o que gera um custo anormal aos serviços de saúde.

Da mesma forma, as sociedades americana e europeia emitiram em 2014 um consenso ${ }^{5}$ sobre a avaliaçáo dos pacientes durante e após a terapia antineoplásica. Nesse documento, além de uma explanação detalhada sobre os métodos de imagem usados hoje em dia no acompanhamento desses doentes, foi criada uma rotina orientando as comunidades médicas sobre o melhor seguimento dos pacientes submetidos a terapias cardiotóxicas tipo I (representada pelos antraciclínicos) e tipo II cujo principal quimioterápico é o trastuzumabe. Assim, identificam-se fluxogramas que sugerem acompanhamento cardiológico naqueles pacientes com queda da FE para abaixo de $53 \%$, ou queda do strain e aumento de troponinas no caso daqueles submetidos a antraciclínicos a cada seis meses no primeiro ano. Já para os pacientes que serão submetidos ao trastuzumabe, sugere-se uma avaliação basal de ecocardiograma, strain ou troponina com suas medidas seriadas a cada três meses.

Há alguns escores de avaliação de fatores de risco e de seguimento propostos por alguns serviços como, por exemplo, o da Clínica Mayo que, em 2016, sugeriu uma graduação de risco. Esta varia conforme os fatores de risco do paciente e da QT proposta, promovendo pontuaçóes de acordo com cada medicação e fator de risco, graduando-os em riscos para o desenvolvimento de CDTX. Assim, os pacientes sofrerão avaliações mais frequentes conforme as pontuaçóes atingidas (Quadro 1) ${ }^{4}$.

Existem outros métodos de estratificação mais simples propostos por outras sociedades, como a Europeia de Cardiologia 5 que, em 2016, publicou um consenso que enfatiza a importância da identificação dos fatores de risco com relaçấo à CDTX causada por antraciclinas (doxorrubicina, epirrubicina), por inibidores de fator de crescimento tumoral VEGF-1 (anticorpos representados pelo bevacizumabe e inibidores de tirosina-quinase exemplificados por sunitinibe, pazopanibe, sorafenibe e dasatinibe), inibidores de Her-2 (trastuzumabe) e os métodos de acompanhamento por meio de biomarcadores e exames de imagem (preferencialmente o mesmo) que podem variar entre ecocardiograma, imagem nuclear magnética (MUGA) e ressonância nuclear magnética (RNM). Além disso, também se utilizam biomarcadores (troponina ou BNP) na tentativa de se detectarem lesóes cardíacas subclínicas e melhor manejo desse paciente com risco mais elevado de CDTX 5 .

Com relação aos antraciclínicos, não fica clara a frequência de acompanhamento com exames de imagem e biomarcadores, apenas que sejam feitos no início e ao fim do tratamento 5 .

Com relação aos inibidores de Her-2, de acordo com a sociedade europeia, deve haver um monitoramento mais intensivo com exames de imagem a cada três meses durante e após o término do tratamento. Muitos estudos revelaram uma melhora na detecçáo precoce da queda da FE quando as troponinas e o ECOTT strain eram usados a cada três meses durante o tratamento com o trastuzumabe. Dada a variabilidade de tempo com que a disfunção causada por esse quimioterápico pode ocorrer, sugere-se que a medida 
Quadro 1. Estimativa de risco e monitoramento associado à disfunção ventricular

Fatores de risco
relacionados ao
paciente
1 ponto para cada
fator de risco
Idade (<15 anos ou
$>65$ anos)

Sexo feminino

Hipertensão

\begin{tabular}{l} 
Diabetes mellitus \\
Aterosclerose (DAC, \\
DCV, DAOP) \\
Doença cardíaca \\
prévia ou \\
insuficiência cardíaca \\
Uso prévio de \\
antraciclínicos \\
RxT mediastinal ou \\
torácica prévias \\
\hline ER de cardiotoxicidade
\end{tabular}

Fatores de risco de acordo com o quimioterápico + número de fatores de risco relacionados ao paciente

ER>6: muito alto; ER 5-6: alto risco; ER 3-4:

intermediário; ER 1-2: baixo; ER 0: muito baixo

Recomendações de monitoramento da Clínica

Mayo

ER > 6: ECOTT strain antes de cada ciclo, ao fim da

QT, 3, 6 e 12 meses após. Opcional ECG e troponina com o eco durante a QT

ER 5-6: ECOTT strain a cada 3 ciclos ao fim da QT, 3, 6 e 12 meses após. Opcional ECG e troponina com o ECOTT durante a QT

ER 3-4: ECOTT strain na metade dos ciclos, 3 e 6 meses ao fim da QT e 12 meses após. Opcional ECG e troponina com o ECO durante a QT

ER 1-2: Opcional ECOTT strain e/ou ECG ao fim do tratamento

ER 0: Sem necessidade de exames

Legendas: $\mathrm{DAC}=$ Doença arterial coronariana; $\mathrm{DCV}=$ Doença cerebrovascular; $\mathrm{DAOP}=$ Doença arterial periférica obstrutiva; $\mathrm{ECG}=$ Eletrocardiograma; $\mathrm{ECOTT}=$ Ecocardiograma transtorácico; ER = Escores de risco; $\mathrm{QT}$ = Quimioterapia; RxT = Radioterapia; Strain = técnica realizada durante o ecocardiograma para avaliar grau de deformidade miocárdica.

de troponina seja realizada a cada ciclo em pacientes com risco de base mais elevado.

A mesma sociedade também preconiza que aqueles que recebem inibidores de VEGF-1 sejam monitorados nas primeiras duas a quatro semanas do início do tratamento e seja feita uma revisão periódica da função cardíaca após seu término. Esta, no entanto, não ficou bem definida quanto ao melhor momento. Frequentemente, considera-se uma revisão a cada seis meses até estabilizaçáo da FE quando esta é afetada 5 .

Por fim, essa mesma vertente adverte que se deve realizar ao menos um ecocardiograma de base e, para os pacientes de baixo risco (ECOTT de base normal e ausência de fatores de risco), o acompanhamento para aqueles que recebem anti-Her-2 seja realizado a cada quatro ciclos ou após uma dose cumulativa de $200 \mathrm{mg} / \mathrm{m}^{2}$ de doxorrubicina ou equivalentes.

Dessa maneira, nota-se que, mesmo entre os consensos, há certa dificuldade em se estabelecer os pacientes com maior risco de desenvolver CDTX e a frequência ideal de monitorização de ecocardiograma e de biomarcadores, assim como o melhor marcador para seguimento e acompanhamento dos pacientes que, porventura, evoluem com insuficiência cardíaca ao longo do tratamento.

\section{CONCLUSÃO}

Precisa-se de um "escore de risco" para predizer a CDTX, não havendo até o presente momento um marcador específico para isso, mas sim um conjunto de vários deles que parecem ser capazes de detectar lesóes subclínicas. Biomarcadores como troponinas e BNP e ecocardiograma com strain têm demonstrado potencial capacidade de prediçáo em pacientes que desenvolverão lesôes miocárdicas de forma mais precoce.

\section{CONTRIBUIÇÕES}

Os autores contribuíram igualmente de forma substancial em todas as etapas do trabalho e aprovaram a versão final a ser publicada.

\section{DECLARAÇÃO DE CONFLITO DE INTERESSES}

Nada a declarar.

\section{FONTES DE FINANCIAMENTO}

Não há.

\section{REFERÊNCIAS}

1. Plana JC, Galderisi M, Barac A, et al. Expert consensus for multimodality imaging evaluation of adult patients during and after cancer therapy: a report from the american society of echocardiography and the european association of cardiovascular imaging. J Am Soc 
Echocardiogr. 2014;27(9):911-39. doi: https://doi. org/10.1016/j.echo.2014.07.012

2. Miller KD, Siegel RL, Linc CC, et al. Cancer treatment and survivorship statistics, 2016. CA Cancer J Clin. 2016;66(4):271-89. doi: https://doi.org/10.3322/ caac. 21349

3. Finet JE. Management of heart failure in cancer patients and cancer survivors. Heart Fail Clin. 2017;13(2):25388. doi: https://doi.org/10.1016/j.hfc.2016.12.004

4. Barros-Gomes S, Herrmann J, Mulvagh SL, et al. Rationale for setting up a cardio-oncology unit: our experience at Mayo Clinic. Cardio-Oncology. 2016;2:5. doi: https://doi.org/10.1186/s40959-016-0014-2

5. Zamorano JL, Lancellotti P, Rodriguez Muñoz D, et al. 2016 ESC Position Paper on cancer treatments and cardiovascular toxicity developed under the auspices of the ESC Committee for Practice Guidelines: the task force for cancer treatments and cardiovascular toxicity of the European Society of Cardiology (ESC). Eur Heart J. 2016;37(36):2768-2801. doi: https://doi.org/10.1093/ eurheartj/ehw211

6. Perez EA, Koehler M, Byrne J, et al. Cardiac safety of lapatinib: pooled analysis of 3689 patients enrolled in clinical trials. Mayo Clin Proc. 2008;83(6):679-86. doi: https://doi.org/10.4065/83.6.679

7. Daugaard G, Lassen U, Bie P, et al. Natriuretic peptides in the monitoring of anthracycline induced reduction in left ventricular ejection fraction. Eur J Heart Fail. 2005;7(1):87-93. doi: https://doi.org/10.1016/j. ejheart.2004.03.009

8. Jurcut R, Wildiers H, Ganane J, et al. Detection and monitoring of cardiotoxicity-what does modern cardiology offer?. Support Care Cancer. 2008;16(5):43745. doi: https://doi.org/10.1007/s00520-007-0397-6

9. Cardinale D, Sandri MT, Colombo A, et al. Prognostic value of troponin I in cardiac risk stratification of cancer patients undergoing high-dose chemotherapy. Circulation. 2004;109(22):2749-54. 96. doi: https:// doi.org/10.1161/01.CIR.0000130926.51766.CC

10. Sawaya H, Sebag IA, Plana JC, et al. Assessment of echocardiography and biomarkers for the extended prediction of cardiotoxicity in patients treated with anthracyclines, taxanes, and trastuzumab. Circ Cardiovasc Imaging. 2012;5(5):596-603. doi: https:// doi.org/10.1161/CIRCIMAGING.112.973321 